PROCEEDINGS OF THE

AMERICAN MATHEMATICAL SOCIETY

Volume 140, Number 6, June 2012, Pages 2103-2116

S 0002-9939(2011)11082-7

Article electronically published on October 20, 2011

\title{
LINEAR OPERATORS WITH WILD DYNAMICS
}

\author{
JEAN-MATTHIEU AUGÉ
}

(Communicated by Thomas Schlumprecht)

\begin{abstract}
If $X$ is a separable infinite-dimensional Banach space, we construct a bounded and linear operator $R$ on $X$ such that

$$
A_{R}=\left\{x \in X,\left\|R^{t} x\right\| \rightarrow \infty\right\}
$$

is not dense and has a non-empty interior with the additional property that $R$ can be written $I+K$, where $I$ is the identity and $K$ is a compact operator. This answers two recent questions of Hájek and Smith.
\end{abstract}

\section{INTRODUCTION}

Let $X$ be a Banach space (we will sometimes distinguish the real and complex cases) and $R$ be a bounded linear operator on $X$. For $x$ in $X$, let

$$
O_{R}(x)=\left\{R^{t} x, t \geqslant 0\right\}
$$

be the orbit of $x$ under the action of $R$. If there exists $x \in X$ such that $O_{R}(x)$ is dense in $X, R$ is called a hypercyclic operator (and $x$ a hypercyclic vector for $R$ ). This class of operator has been intensively studied in the last two decades; see BM for a nice survey on this topic. Furthermore, the orbits are connected with the invariant subset problem which asks if there exists an operator on $X$ with a non-trivial invariant closed subset. Indeed, $R$ does not have any trivial invariant closed subset if and only if for each $x \neq 0, O_{R}(x)$ is dense in $X$. In the space $\ell^{1}$, Read $[\mathrm{R}]$ constructed such an operator. However, in the Hilbert space, the problem is still open. Here, we will be interested in the orbits which tend to infinity. The following is our main result.

Theorem 1.1. Let $X$ be an infinite-dimensional separable, real or complex, Banach space. There exists a linear continuous operator $R$ on $X$ such that if we put

$$
A_{R}=\left\{x \in X,\left\|R^{t} x\right\| \rightarrow \infty\right\} \text { and } B_{R}=\left\{x \in X, \underline{\lim }\left\|R^{t} x-x\right\|=0\right\},
$$

A) $A_{R}$ and $B_{R}$ have non-empty interior and $\left\{A_{R}, B_{R}\right\}$ form a partition of $X$;

$B) R$ can be written $I+K$, with $K$ a compact operator.

As a direct consequence, we obtain the following more general result.

Corollary 1.2. The conclusion of Theorem 1.1 remains true if one only assumes that $X$ has a closed and separable subspace of infinite dimension which is complemented in $X$.

Received by the editors November 18, 2010 and, in revised form, February 11, 2011. 2010 Mathematics Subject Classification. Primary 47A05; Secondary 47A15, 47A16.

Key words and phrases. Orbits of operators, compact operators.

(C)2011 American Mathematical Society Reverts to public domain 28 years from publication 
Proof. We write $X=X_{0} \oplus Y$, where $X_{0} \subset X$ is a closed separable Banach space of infinite dimension and $Y$ is a closed subspace of $X$. We then consider $R=R_{0} \oplus I$, where $R_{0}$ is an operator on $X_{0}$ which satisfies Theorem 1.1. Since $R^{t}=R_{0}^{t} \oplus I$, we get $A_{R}=A_{R_{0}}+Y, B_{R}=B_{R_{0}}+Y$, and it is clear that $R$ has the required properties.

Theorem 1.1 is motivated by a conjecture of Prăjitură $[\mathbb{P}$ which was recently solved negatively by Hájek and Smith [HS]:

Conjecture 1.3. Let $R$ be an operator on a Banach space. Then $A_{R}$ is either empty or dense.

Hájek and Smith showed part A of Theorem 1.1 when $X$ has a symmetric basis. Therefore, they asked if the theorem could be true for a space which has only an unconditional basis (which is a weaker property than having a symmetric basis). We show that in fact no hypothesis of basis existence is necessary. Furthermore, a second question they asked was: is it possible to choose the operator $R$ of the form $I+K$, with $K$ a compact operator? The reason for this question is that they observed that for a compact operator $K$ and more generally, for a strictly singular operator $S$ (that is, an operator which is not an isomorphism when restricted to any infinite-dimensional subspace), $A_{S}$ was either empty or dense. On the other hand, there exist some spaces with very few operators: Gowers and Maurey GM] constructed a Banach space where each operator has the form $\lambda I+S$, with $S$ strictly singular and, even more recently, Argyros and Haydon [AH] found a space where each operator has the form $\lambda I+K$, with $K$ compact. Thus, if some operators satisfying part A of Theorem 1.1 exist on any separable Banach space, it must be true that they can take the form $\lambda I+K$, and we show that it is indeed possible.

Note that if $\left(\left\|R^{t}\right\|\right)_{t \geqslant 0}$ is unbounded, by the uniform boundedness principle,

$$
A_{R}^{\prime}=\left\{x \in X,\left(\left\|R^{t} x\right\|\right)_{t \geqslant 0} \text { is unbounded }\right\}
$$

is a dense $G_{\delta}$ in $X$. Thus, for any operator $R, A_{R}^{\prime}$ is either dense or empty. However, this does not show anything for $A_{R}$ since there exist some operators such that $\left\|R^{t}\right\| \rightarrow \infty$, but for all $x,\left\|R^{t} x\right\| \nrightarrow \infty$. For example, it is the case for the following weighted backward shift $\left[\mathrm{HS}\right.$ ] defined on $\ell^{p}(1 \leqslant p<\infty)$ :

$$
B e_{i}=\left\{\begin{array}{l}
\left(\frac{i}{i-1}\right)^{\frac{1}{p}} e_{i-1} \text { if } i>1, \\
0 \text { if } i=1 .
\end{array}\right.
$$

However, Müller and Vršovský [MV] showed that if $\left\|R^{t}\right\|$ grows fast enough, namely if $\sum_{k=1}^{\infty} \frac{1}{\left\|R^{k}\right\|}<\infty$, then $A_{R}$ is dense.

Let us explain the organization of this paper. Section 2 is devoted to a preliminary result: we prove a separating lemma in finite dimension (which might be of independent interest). We then use it in section 3 to construct an operator satisfying Theorem 1.1 (we treat the complex and real cases separately). In section 4, we present a kind of version of the operator constructed by Hájek and Smith with a slightly simplified proof. In section 5, we make some comments on the two operators and we prove that in fact there are many operators which fail Prăjitură's conjecture.

Let us settle some notation: if $X$ is a Banach space, we denote by $\mathcal{L}(X)$ the set of linear and bounded operators on $X$, by $\mathcal{G} \mathcal{L}(X) \subset \mathcal{L}(X)$ the set of invertible 
operators and by $\mathcal{A}_{X}$ the set of operators which satisfy part A of Theorem 1.1. Until the end of the paper $X$ will always be assumed to be infinite dimensional (if $X$ is finite dimensional, Prăjitură's conjecture is true; see [HS]).

\section{An ASYMPtOTIC SEPARATING LEMMA IN FINITE DIMENSION}

Here $d \geqslant 2$ is an integer and $\mathbb{K}=\mathbb{R}$ or $\mathbb{C}$.

Proposition 2.1. Let $H \neq \emptyset$ be a closed subset of $\mathbb{K}^{d}$ such that $H$ is a union of linear hyperplanes. Then there exists a sequence of linear forms $\left(f_{n}\right)$ on $\mathbb{K}^{d}$ such that

1) For all $x \notin H, \lim \left|f_{n}(x)\right|=\infty$.

2) For all $x \in H, \underline{\lim }\left|f_{n}(x)\right|=0$.

We will do the proof in the real case. Let us denote by $S=S_{\mathbb{R}^{d}}$ the unit sphere of $\mathbb{R}^{d}$ and put $C=S \cap H$. We first prove the following lemma.

Lemma 2.2. There exists a constant $K>0$ and a sequence $\left(u_{n}\right) \subset C$ such that for each $x \in C$ there exists a sequence of integers $\left(p_{n}\right)$ which goes to infinity and satisfies for each $n, p_{n} \leqslant n$ and $\left\|x-u_{p_{n}}\right\| \leqslant \frac{K}{n^{1 / d-1}}$.

Proof of Lemma 2.2. There exists $L>0$ such that for each $k$ we can find a $2^{-k}$ net $H_{k}$ of $C$ with $\left|H_{k}\right| \leqslant L\left(2^{k}\right)^{d-1}$, where $\left|H_{k}\right|$ denotes the number of elements of $H_{k}$. The sequence $\left(u_{n}\right)$ is obtained by enumerating first the elements of $H_{1}$, then all the elements of $H_{2}$, and so on. Let us fix $x \in C$ and check the property of the lemma. For $n \in \mathbb{N}$, there exists an integer $k$ such that $\left|H_{1}\right|+\ldots+\left|H_{k}\right| \leqslant n<$ $\left|H_{1}\right|+\ldots+\left|H_{k}\right|+\left|H_{k+1}\right|$. In particular, we get

$$
n \leqslant L \sum_{i=1}^{k+1}\left(2^{d-1}\right)^{i}=2^{d-1} L \frac{\left(2^{d-1}\right)^{k+1}-1}{2^{d-1}-1} \leqslant C_{d} 2^{k(d-1)},
$$

where $C_{d}>0$ only depends on $d$. By the definition of $H_{k}$, there exists an element $u_{p_{n}} \in H_{k}$ such that $\left\|x-u_{p_{n}}\right\| \leqslant 2^{-k} \leqslant\left(\frac{C_{d}}{n}\right)^{1 / d-1}$. Since $k-1 \leqslant\left|H_{1}\right|+\ldots+\left|H_{k-1}\right|<$ $p_{n} \leqslant\left|H_{1}\right|+\ldots+\left|H_{k}\right|, p_{n} \leqslant n, \lim p_{n}=\infty$ and the lemma is shown.

Proof of Proposition 2.1. For each $n$, we can select a linear form $f_{n}$ such that $f_{n}\left(u_{n}\right)=0, \operatorname{Ker} f_{n} \subset H$ and $\left\|f_{n}\right\|=n^{1 / 2(d-1)}$. If $x \notin H$, then

$$
\left|f_{n}(x)\right|=\left\|f_{n}\right\| d\left(x, \operatorname{Ker} f_{n}\right) \geqslant\left\|f_{n}\right\| d(x, H) \geqslant n^{1 / 2(d-1)} d(x, H) .
$$

Thus we have 1). For 2), we may assume that $x \neq 0$ and apply the lemma with $y=\frac{x}{\|x\|} \in C$. We obtain a sequence $\left(p_{n}\right)$ with $p_{n} \leqslant n, \lim p_{n}=\infty$ and $\left\|y-u_{p_{n}}\right\| \leqslant$ $\frac{K}{n^{1 / d-1}}$. For each $n$ we have

$$
\left|f_{p_{n}}(x)\right|=\|x\|\left|f_{p_{n}}\left(y-u_{p_{n}}\right)\right| \leqslant\left\|f_{p_{n}}\right\|\left\|y-u_{p_{n}}\right\|\|x\| \leqslant \frac{K}{n^{1 / 2(d-1)}}\|x\|,
$$

which proves 2) and achieves the proof.

For the complex case, the proof is almost the same. The only difference is that for $\epsilon>0$ the entropy of the unit sphere is of the order of $\frac{1}{\epsilon^{2 d-1}}$, which gives a different estimate of $\left\|x-u_{p_{n}}\right\|$ in the lemma, so we also have to change the exponent in $\left\|f_{n}\right\|$ in the proof of the proposition. 


\section{Construction of operators}

3.1. Complex case. In this sub-section, we prove the complex version of Theorem 1.1

Theorem 3.1. Let $X$ be a separable complex Banach space. Then, there exists $R \in \mathcal{A}_{X}$ such that $R-I$ is nuclear.

Recall that an operator $N$ is nuclear if there exist some continuous linear functionals $x_{k}^{*}$ and some vectors $x_{k}$ such that $N$ can be written as $N=\sum_{k=1}^{\infty} x_{k} \otimes x_{k}^{*}$ with $\sum_{k=1}^{\infty}\left\|x_{k}^{*}\right\|\left\|x_{k}\right\|<\infty$, where $x_{k} \otimes x_{k}^{*}$ is the rank one operator defined by

$$
x_{k} \otimes x_{k}^{*}(x)=\left\langle x_{k}^{*}, x\right\rangle x_{k} .
$$

These operators are obviously compact, being limits of finite rank operators.

To construct our operator, we will use the following theorem which says that a separable Banach space supports a sequence $\left(e_{n}\right)_{n \geqslant 1}$ which behaves more or less like a Schauder basis $\mathrm{OP}$.

Theorem 3.2. If $X$ is a separable Banach space and $\epsilon>0$, one can find sequences $\left(e_{n}, e_{n}^{*}\right) \subset X \times X^{*}$ such that:

i) $\operatorname{span}\left(e_{n}, n \geqslant 1\right)$ is dense in $X$;

ii) $\left\langle e_{n}^{*}, e_{m}\right\rangle=\delta_{n, m}$, where $\delta_{n, m}=0$ if $n \neq m$ and 1 if $n=m$;

iii) $\sup \left\|e_{n}\right\|\left\|e_{n}^{*}\right\| \leqslant 1+\epsilon$.

For our case, we will not need the precise estimate with $\epsilon$. However, observe that by replacing $e_{n}$ by $e_{n} /\left\|e_{n}\right\|$ and $e_{n}^{*}$ by $\left\|e_{n}\right\| e_{n}^{*}$, we can suppose that $\left\|e_{n}\right\|=1$, $K=\sup \left\|e_{n}^{*}\right\|<\infty$ and properties i) and ii) of Theorem 3.2 are still satisfied. We then consider a closed subset $F \subset \operatorname{span}\left(e_{1}, e_{2}\right)$ which is a union of complex lines passing through 0 and the sequence $\left(f_{k}\right)$ of linear forms associated on $\operatorname{span}\left(e_{1}, e_{2}\right)$ in the asymptotic separating lemma (we just use the particular case of the lemma $d=2)$. Let us denote by $P$ the projection defined on $X$ onto $\operatorname{span}\left(e_{1}, e_{2}\right)$ by

$$
P x=\left\langle e_{1}^{*}, x\right\rangle e_{1}+\left\langle e_{2}^{*}, x\right\rangle e_{2} .
$$

$P$ is continuous with $\|P\| \leqslant 2 K$. Then, we put $\lambda_{1}=\lambda_{2}=1$ and for $k \geqslant 3$, $\lambda_{k}=e^{\frac{i \pi}{m_{k}}}$, where $\left(m_{k}\right)$ is a sequence of positive integers such that $m_{k} \mid m_{k+1}$ and which goes very fast to infinity, namely,

$$
\sum_{k=3}^{\infty} \frac{m_{k-2}}{m_{k-1}}\left\|f_{k}\right\|<\infty \text { and } m_{k} \geqslant 15 m_{k-1} .
$$

We denote by $c_{00}$ the subspace generated by the sequence $\left(e_{n}\right)_{n \geqslant 1}$. For $x \in c_{00}$, $x=\sum_{k=1}^{n} x_{k} e_{k}$, we set

$$
S x=\sum_{k=1}^{n} \lambda_{k} x_{k} e_{k}
$$

This defines a bounded operator $S$ on $c_{00}$. Indeed, for $x=\sum_{k=1}^{n} x_{k} e_{k} \in c_{00}$,

$$
\|S x\| \leqslant \sum_{k=1}^{n}\left|\lambda_{k}-1\right|\left\|x_{k} e_{k}\right\|+\|x\| \leqslant\left(K \sum_{k=1}^{\infty}\left|\lambda_{k}-1\right|+1\right)\|x\|,
$$

and by our assumption on $\left(m_{k}\right)$, it is clear that $\sum_{k=1}^{\infty}\left|\lambda_{k}-1\right|<\infty$. Hence, $S$ extends to a bounded operator on $X$ (because $c_{00}$ is dense in $X$ ). We still denote this operator by $S$. 
Remark 3.3. Note that if $\left(e_{k}\right)$ is a normalized Schauder basis and then if $x=$ $\sum_{k=1}^{\infty} x_{k} e_{k}, S$ is explicitly given by

$$
S x=\sum_{k=1}^{\infty} \lambda_{k} x_{k} e_{k} .
$$

For $x \in X$, we now define an operator $R$ by

$$
R x=S x+\sum_{k=3}^{\infty} \frac{1}{m_{k-1}} f_{k}(P x) e_{k} .
$$

Note that the second sum is a bounded operator because $\sum_{k=3}^{\infty} \frac{1}{m_{k-1}}\left\|f_{k}\right\|<\infty$. Thus $R$ is itself bounded. If we set

$$
\tilde{A}_{R}=\{x \in X, P x \notin F\} \text { and } \tilde{B}_{R}=\{x \in X, P x \in F\},
$$

then we will show that $A_{R}=\tilde{A}_{R}$ and $B_{R}=\tilde{B}_{R}$, so $R$ will be the required operator (provided the relative interior of $F$ in $\operatorname{span}\left(e_{1}, e_{2}\right)$ is non-empty and $\left.F \neq \operatorname{span}\left(e_{1}, e_{2}\right)\right)$. Since $\lambda_{k}^{2 m_{k}}=1$ and more generally $\lambda_{k}^{2 m_{n}}=1$ for $n \geqslant k$ (by the hypothesis of divisibility of the $m_{k}$ ), the operator $S$ has the following property.

Lemma 3.4. For $x \in X, \lim _{n \rightarrow \infty} S^{2 m_{n}} x=x$.

Proof. We first show that the sequence $\left(\left\|S^{2 m_{l}}\right\|\right)$ is bounded. If we fix $x=\sum_{k=1}^{n} x_{k} e_{k}$ such that $\|x\|=1$, we have

$$
\begin{aligned}
\left\|S^{2 m_{l}} x-x\right\| & =\left\|\sum_{k=1}^{n}\left(\lambda_{k}^{2 m_{l}}-1\right) x_{k} e_{k}\right\| \\
& =\left\|\sum_{k=l+1}^{n}\left(\lambda_{k}^{2 m_{l}}-1\right) x_{k} e_{k}\right\| \text { because } \lambda_{k}^{2 m_{l}}=1 \text { for } l \geqslant k \\
& \leqslant K \sum_{k=l+1}^{n}\left|\lambda_{k}^{2 m_{l}}-1\right| \\
& \leqslant 2 K \sum_{k=l+1}^{\infty} \frac{2 \pi m_{l}}{m_{k}} \text { because }\left|e^{z}-1\right| \leqslant 2|z| \text { for }|z| \leqslant 1 / 2 \\
& \leqslant K \sum_{k=1}^{\infty} \frac{4 \pi m_{k-1}}{m_{k}} .
\end{aligned}
$$

Hence $\left(\left\|S^{2 m_{l}} x\right\|\right)$ is bounded by $1+K \sum_{k=1}^{\infty} \frac{4 \pi m_{k-1}}{m_{k}}<\infty$ whenever $x \in c_{00}$, $\|x\|=1$. By density of $c_{00}$ in $X$, this implies that the sequence $\left(\left\|S^{2 m_{l}}\right\|\right)$ is bounded. To see the claim of the lemma, fix $x \in X, \epsilon>0$ and $y \in c_{00}$ such that $\|y-x\| \leqslant \epsilon$, we have

$$
\begin{aligned}
\left\|S^{2 m_{l}} x-x\right\| & =\left\|S^{2 m_{l}}(x-y)+\left(S^{2 m_{l}} y-y\right)+(y-x)\right\| \\
& \leqslant \sup \left\|S^{2 m_{l}}\right\| \epsilon+\left\|S^{2 m_{l}} y-y\right\|+\epsilon .
\end{aligned}
$$

Since for large enough $l, S^{2 m_{l}} y=y$. We have the conclusion.

We now make explicit the iterates of $R$. 
Lemma 3.5. For $x \in X$ and $t \geqslant 1$, we have

$$
R^{t} x=S^{t} x+\sum_{k=3}^{\infty} \frac{\lambda_{k, t}}{m_{k-1}} f_{k}(\text { Px }) e_{k}, \text { where } \lambda_{k, t}=\sum_{l=0}^{t-1} \lambda_{k}^{l} .
$$

Proof. The statement is true for $t=1$. We proceed by induction and suppose it is true for $t$. We have

$$
\begin{aligned}
R^{t+1} x=R\left(R^{t} x\right) & =R\left(S^{t} x\right)+R\left(\sum_{k=3}^{\infty} \frac{\lambda_{k, t}}{m_{k-1}} f_{k}(P x) e_{k}\right) \\
& =S^{t+1} x+\sum_{k=3}^{\infty} \frac{1}{m_{k-1}} f_{k}\left(P S^{t} x\right) e_{k}+\sum_{k=3}^{\infty} \frac{\lambda_{k} \lambda_{k, t}}{m_{k-1}} f_{k}(P x) e_{k} \\
& +\sum_{k=3}^{\infty} \frac{1}{m_{k-1}} f_{k}(P y) e_{k},
\end{aligned}
$$

where $y=\sum_{l=3}^{\infty} \frac{\lambda_{l, t}}{m_{l-1}} f_{l}(P x) e_{l}$, so $P y=0$. Since $\lambda_{1}=\lambda_{2}=1$, we have $P S^{t} x=P x$, and since $\lambda_{k} \lambda_{k, t}+1=\lambda_{k, t+1}$, we deduce the formula for $t+1$.

We now study the behaviour of $\lambda_{k, t}$. We have the following easy fact.

Fact 3.6. i) For all $k, t \geqslant 1,\left|\lambda_{k, t}\right| \leqslant t$.

ii) $\lambda_{k, 2 m_{n}}=0$ for $n \geqslant k$.

iii) For $m_{k-1} \leqslant t \leqslant m_{k},\left|\lambda_{k, t}\right| \geqslant \frac{2}{\pi} m_{k-1}$.

Proof. i) is just the triangle inequality. ii) is obvious since $\lambda_{k, t}$ can be computed easily as a geometric sum:

$$
\lambda_{k, t}=\frac{e^{\frac{i \pi t}{m_{k}}}-1}{e^{\frac{i \pi}{m_{k}}}-1} .
$$

For iii), we get

$$
\left|\lambda_{k, t}\right|=\frac{\left|\sin \left(\frac{\pi t}{2 m_{k}}\right)\right|}{\left|\sin \left(\frac{\pi}{2 m_{k}}\right)\right|} .
$$

The desired lower bound follows from the inequalities $\sin (y) \geqslant \frac{2}{\pi} y$ for $y \in\left[0, \frac{\pi}{2}\right]$ and $|\sin (y)| \leqslant|y|$ for $y \in \mathbb{R}$.

We can now pass to the proof of the main theorem. Let $x \in c_{00} \cap \tilde{A}_{R}, x=$ $\sum_{k=1}^{n} x_{k} e_{k}$. Fix $k \geqslant 3$. We set $\delta_{k \leqslant n}=1$ if $k \leqslant n$ and $\delta_{k \leqslant n}=0$ if $k>n$. We have

$$
\begin{aligned}
K\left\|R^{t} x\right\| \geqslant\left|\left\langle e_{k}^{*}, R^{t} x\right\rangle\right| & =\left|\lambda_{k}^{t}\left\langle e_{k}^{*}, x\right\rangle \delta_{k \leqslant n}+\frac{\lambda_{k, t}}{m_{k-1}} f_{k}(P x)\right| \\
& \geqslant\left|\frac{\lambda_{k, t}}{m_{k-1}} f_{k}(P x)\right|-\left|\lambda_{k}^{t}\left\langle e_{k}^{*}, x\right\rangle \delta_{k \leqslant n}\right| \\
& \geqslant\left|\frac{\lambda_{k, t}}{m_{k-1}} f_{k}(P x)\right|-K\|x\| .
\end{aligned}
$$

We claim that $\tilde{A}_{R} \cap c_{00}$ is dense in $\tilde{A}_{R}$. Since the last inequality involves only continuous functions, it stays true for $x \in \tilde{A}_{R}$ and for $k \geqslant 3, t \geqslant 1$. Now, let us fix $k \geqslant 1$ and $m_{k-1} \leqslant t \leqslant m_{k}$. Using the last inequality and the previous fact, we get

$$
\left\|R^{t} x\right\| \geqslant\left|\frac{\lambda_{k, t}}{K m_{k-1}} f_{k}(P x)\right|-\|x\| \geqslant \frac{2}{\pi K}\left|f_{k}(P x)\right|-\|x\| .
$$


This proves that $\left\|R^{t} x\right\| \rightarrow \infty$ whenever $x \in \tilde{A}_{R}$, because by the separating lemma $\left|f_{k}(P x)\right| \rightarrow \infty$. For the proof of the claim, if $x \in \tilde{A}_{R}$, there exists $\left(z_{k}\right) \subset c_{00}$ such that $x=\lim z_{k}$. Write $z_{k}=z_{1, k} e_{1}+z_{2, k} e_{2}+y_{k}$ with $y_{k} \in \operatorname{span}\left(e_{l}, l \geqslant 3\right)$. If $P x=x_{1} e_{1}+x_{2} e_{2}$, the sequence $\tilde{z_{k}}=x_{1} e_{1}+x_{2} e_{2}+y_{k}$ has the required properties.

Now, let $x \in \tilde{B}_{R}$. Then there exists a subsequence $\left(k_{n}\right)$ such that $\left|f_{k_{n}}(P x)\right|$ goes to 0 . Write

$$
\begin{aligned}
\left\|\sum_{k=3}^{\infty} \frac{\lambda_{k, 2 m_{k_{n}-1}}}{m_{k-1}} f_{k}(P x) e_{k}\right\| \leqslant & \sum_{k=3}^{k_{n}-1} \frac{\left|\lambda_{k, 2 m_{k_{n}-1}}\right|}{m_{k-1}}\left|f_{k}(P x)\right|+\frac{\left|\lambda_{k_{n}, 2 m_{k_{n}-1}}\right|}{m_{k_{n}-1}}\left|f_{k_{n}}(P x)\right| \\
& +\sum_{k=k_{n}+1}^{\infty} \frac{\left|\lambda_{k, 2 m_{k_{n}-1}}\right|}{m_{k-1}}\left|f_{k}(P x)\right| .
\end{aligned}
$$

The first sum is equal to 0 according to the previous fact. Using the fact again, we see that the second term goes to 0 because $\frac{\left|\lambda_{k_{n}, 2 m_{k_{n}-1}}\right|}{m_{k_{n}-1}} \leqslant 2$. For the last sum, $k \geqslant k_{n}+1$ so $k_{n}-1 \leqslant k-2$, and $m_{k_{n}-1} \leqslant m_{k-2}$. Hence

$$
\begin{aligned}
\sum_{k=k_{n}+1}^{\infty} \frac{\left|\lambda_{k, 2 m_{k_{n}-1}}\right|}{m_{k-1}}\left|f_{k}(P x)\right| & \leqslant\left(\sum_{k=k_{n}+1}^{\infty} \frac{2 m_{k_{n}-1}}{m_{k-1}}\left\|f_{k}\right\|\right)\|P\|\|x\| \\
& \leqslant\left(\sum_{k=k_{n}+1}^{\infty} \frac{2 m_{k-2}}{m_{k-1}}\left\|f_{k}\right\|\right)\|P\|\|x\| .
\end{aligned}
$$

Since $\sum_{k \geqslant 1} \frac{m_{k-2}}{m_{k-1}}\left\|f_{k}\right\|<\infty$, the last term goes to 0 . On the other hand, $S^{2 m_{k_{n}-1}} x$ goes to $x$; this shows that $\underline{\lim }\left\|R^{t} x-x\right\|=0$ whenever $x \in \tilde{B}_{R}$.

Finally, let us check the last claim of our theorem. By a density argument we see that for $x \in X$,

Hence

$$
(S-I) x=\sum_{k=3}^{\infty}\left(\lambda_{k}-1\right)\left\langle e_{k}^{*}, x\right\rangle e_{k}
$$

$$
N=R-I=\sum_{k=3}^{\infty} e_{k} \otimes\left(\left(\lambda_{k}-1\right) e_{k}^{*}+\frac{1}{m_{k-1}} f_{k} P\right) .
$$

Since $\sum_{k=3}^{\infty}\left|\lambda_{k}-1\right|<\infty, \sum_{k=3}^{\infty} \frac{1}{m_{k-1}}\left\|f_{k}\right\|<\infty$, and $\left(e_{k}\right)$ and $\left(e_{k}^{*}\right)$ are bounded, $N$ is nuclear and $R=I+N$.

Remark 3.7. The statement of our theorem can be sharpened: it is apparent from the definition of $R$ that for any $\epsilon>0$, one can choose the sequence $\left(m_{k}\right)$ to have $\|R-I\| \leqslant \epsilon$. This proves that $I$ is in the norm closure of $\mathcal{A}_{X}$.

Remark 3.8. Although Corollary 1.2 does not apply to $\ell^{\infty}$, we can see that our example works in $\ell^{\infty}$. We thus conjecture that operators with the properties stated in Theorem 1.1 can be constructed in arbitrary Banach spaces.

3.2. Real case. We now show the version of Theorem 1.1 for real spaces.

Theorem 3.9. Let $X$ be a separable real Banach space. Then, there exists $R \in \mathcal{A}_{X}$ such that $R-I$ is nuclear.

We keep the same notation as in the complex case for $F,\left(f_{k}\right), c_{00},\left(e_{k}\right),\left(e_{k}^{*}\right), K$ and $P$. We define $S$ on $c_{00}$ by the formulas $S e_{1}=e_{1}, S e_{2}=e_{2}$ and for $k \geqslant 2$,

$$
S e_{2 k-1}=\cos \left(\theta_{k}\right) e_{2 k-1}+\sin \left(\theta_{k}\right) e_{2 k} \text { and } S e_{2 k}=-\sin \left(\theta_{k}\right) e_{2 k-1}+\cos \left(\theta_{k}\right) e_{2 k},
$$


where $\theta_{k}=\frac{\pi}{m_{k}}$ and $\left(m_{k}\right)$ satisfy the same hypotheses of growth and divisibility as in the complex case. Thus in the (algebraic) basis $\left(e_{k}\right), S$ is a block diagonal operator built from rotation matrices. We check that $S$ is bounded on $c_{00}$, so it extends to $X$, and we check that $S^{2 m_{n}} x \rightarrow x$. We then define $R$ (the required operator) by

$$
R x=S x+\sum_{k=2}^{\infty} \frac{1}{m_{k-1}} f_{k}(P x) e_{2 k} .
$$

The computation of the iterates of $R$ gives

$$
R^{t} x=S^{t} x+\sum_{k=2}^{\infty} \frac{1}{m_{k-1}} f_{k}(P x) \mu_{k, t}
$$

where $\mu_{k, t}=\left(\sum_{l=0}^{t-1} \cos \left(l \theta_{k}\right)\right) e_{2 k}-\left(\sum_{l=0}^{t-1} \sin \left(l \theta_{k}\right)\right) e_{2 k-1}$. We thus have $\mu_{k, t}=$ $0 \Leftrightarrow \lambda_{k, t}=0,\left\|\mu_{k, t}\right\| \leqslant 2 t$ and considering the functionals $e_{2 k-1}^{*}$ and $e_{2 k}^{*}$,

$$
\left\|\mu_{k, t}\right\| \geqslant \frac{1}{K} \max \left(\left|\sum_{l=0}^{t-1} \cos \left(l \theta_{k}\right)\right|,\left|\sum_{l=0}^{t-1} \sin \left(l \theta_{k}\right)\right|\right) \geqslant \frac{1}{\sqrt{2} K}\left|\lambda_{k, t}\right| .
$$

Thus the inequalities for $\mu_{k, t}$ are the same as $\lambda_{k, t}$ (up to a multiplicative constant), which allows us to follow the previous proof (in the complex case) without other changes.

\section{The construction of Hájek and Smith}

We present a slightly modified version of the example of Hájek and Smith. The following theorem is of course a particular case of our theorem above. However, we will outline some differences between the two operators in the last section.

Theorem 4.1. Let $X$ be a Banach space (real or complex) with a symmetric basis. Then, there exists $R \in \mathcal{A}_{X}$.

Recall that a basis $\left(e_{k}\right)$ is said to be symmetric if for every permutation $\pi$ of the integers, $\left(e_{\pi(k)}\right)$ is a basis and is equivalent to $\left(e_{k}\right)$. If for $x=\sum_{k=1}^{\infty} x_{k} e_{k} \in X$ we set

$$
\|x\|_{0}=\sup _{\left|\lambda_{k}\right| \leqslant 1} \sup _{\pi}\left\|\sum_{k=1}^{\infty} x_{k} \lambda_{k} e_{\pi(k)}\right\|
$$

then $\|\cdot\|_{0}$ is an equivalent norm on $X$ which satisfies for every $\pi$,

$$
\left\|\sum_{k=1}^{\infty} x_{k} e_{\pi(k)}\right\|_{0}=\left\|\sum_{k=1}^{\infty}\left|x_{k}\right| e_{k}\right\|_{0}
$$

and whenever $0 \leqslant x_{k} \leqslant y_{k}$,

$$
\left\|\sum_{k=1}^{\infty} x_{k} e_{k}\right\|_{0} \leqslant\left\|\sum_{k=1}^{\infty} y_{k} e_{k}\right\|_{0}
$$

In the rest of this section, we suppose that our norm $\|\cdot\|$ has the two preceding properties (such a norm is sometimes called a symmetric norm).

In the complex case, our operator $S$ was built with roots of unity; $S$ will now be a cycle operator. As in the original paper of Hájek and Smith, we will prove some finite-dimensional estimates. However, Hájek and Smith make some estimates in 
$\ell^{p}$ and $c_{0}$ and then combine it with a theorem of Tzafriri $[\mathrm{T}$. We will give a direct and short proof of these estimates.

4.1. Local estimates. Let $Z$ be a space of dimension $H \geqslant 1$ with a symmetric basis $\left(e_{1}, \ldots, e_{H}\right)$ such that $H \geqslant 4 m$ ( $m$ and $H$ are positive integers). We define an operator $S$ on $Z$ by $S e_{i}=e_{i+1}$ for $1 \leqslant i \leqslant H-1$ and $S e_{H}=e_{1}$. We will be interested in the behaviour of the sequence $w_{t}=\left(I+\ldots+S^{t-1}\right) w$, where $w=\sum_{i=1}^{m} e_{i}-\sum_{i=m+1}^{2 m} e_{i}$ (this is motivated by the fact that $\left(w_{t}\right)$ will appear in the study of the iterates of the operator in the general case). We have:

Lemma 4.2. i) $w_{H}=0$ and the sequence $\left(w_{t}\right)_{t \geqslant 1}$ is $H$-periodic.

ii) If $2 m \leqslant t \leqslant H-2 m,\left\|w_{t}\right\|$ attains its maximum and

$$
\left\|w_{t}\right\| \geqslant \frac{m}{2}\|w\| .
$$

Proof. i) $S$ is a cycle operator of order $H$. Thus for $1 \leqslant i \leqslant H$, we have $(I+S+$ $\left.\ldots+S^{H-1}\right) e_{i}=\sum_{i=1}^{H} e_{i}$, and if we put $s=\sum_{i=1}^{H} e_{i}$, we get $w_{H}=m s-m s=0$. Moreover, since $S^{H}$ is the identity, we have

$$
w_{t+H}=\left(I+S+\ldots+S^{H-1}\right) w+\left(I+\ldots+S^{t-1}\right) w=\left(I+\ldots+S^{t-1}\right) w=w_{t} .
$$

ii) An easy computation shows that for $2 m \leqslant t \leqslant H-2 m$, w $w_{t}$ has two bumps of height $m$ and of length $2 m-1$, one with positive terms, the other with negative terms:

$$
\begin{aligned}
w_{t}= & e_{1}+2 e_{2}+\ldots+m e_{m}+(m-1) e_{m+1}+\ldots e_{2 m-1} \\
& -\left(e_{t+1}+2 e_{t+2}+\ldots+m e_{t+m}+\ldots e_{t+2 m-1}\right) .
\end{aligned}
$$

Because the norm is symmetric, we can arrange the terms in a decreasing way and cancel the signs; hence

$$
\begin{aligned}
\left\|w_{t}\right\|= & m\left(e_{1}+e_{2}\right)+(m-1)\left(e_{3}+e_{4}+e_{5}+e_{6}\right) \\
& +\ldots+\left(e_{4 m-5}+e_{4 m-4}+e_{4 m-3}+e_{4 m-2}\right) \| .
\end{aligned}
$$

If we keep the coordinates which are superior to $\frac{m}{2}$, we get

$$
\left\|w_{t}\right\| \geqslant \frac{m}{2}\left\|e_{1}+\ldots+e_{2 m}\right\|=\frac{m}{2}\|w\| .
$$

We have also shown that if $u_{t}$ is the decreasing rearrangement of $\left|w_{t}\right|$, then for $2 m \leqslant t \leqslant H-2 m$ and $2 m \leqslant s \leqslant H-2 m, u_{t}=u_{s}$. Another computation shows that for $1 \leqslant s \leqslant 2 m$ and $2 m \leqslant t \leqslant H-2 m, u_{s} \leqslant u_{t}$, and hence that $\left\|w_{s}\right\|=\left\|u_{s}\right\| \leqslant\left\|u_{t}\right\|=\left\|w_{t}\right\|$ because the norm is symmetric. For $H-2 m \leqslant s \leqslant H$, we obtain the same estimates as in the case $1 \leqslant s \leqslant 2 m$. This shows that $\left\|w_{t}\right\|$ attains its maximum for $2 m \leqslant t \leqslant H-2 m$. Below are some graphics of $w_{t}$ for $1 \leqslant t \leqslant 2 m$ that illustrate the inequalities (with $H=30, m=6$ ).

4.2. The operator of Hájek and Smith. We assume that $X$ is a Banach space with a symmetric and normalized basis $\left(e_{n}\right)$ and write

$$
X=\operatorname{span}\left(e_{1}, e_{2}\right) \oplus \overline{\operatorname{span}}\left(e_{n}, n \geqslant 3\right)=\operatorname{span}\left(e_{1}, e_{2}\right) \oplus Y .
$$

Next, we are going to define $S$ on $X$ (we give it the same name as in the finitedimensional case because it will be a sum of these operators on finite blocks). We choose some sequences $\left(H_{k}\right)$ and $\left(m_{k}\right)$ of positive increasing integers such that 


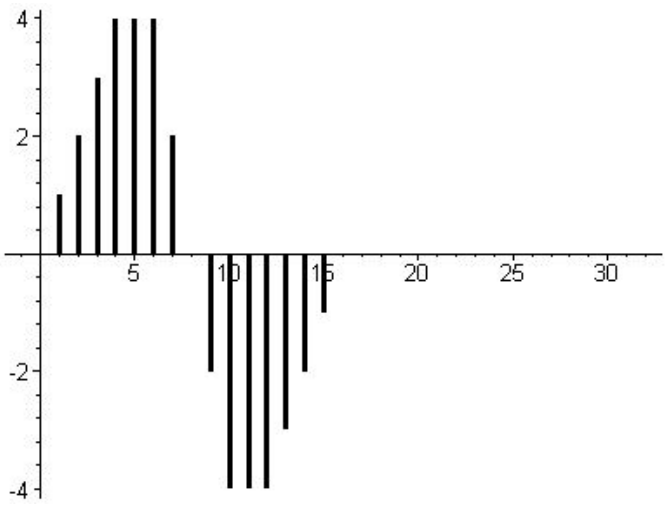

Figure 1. Coordinates of $w_{4}$.

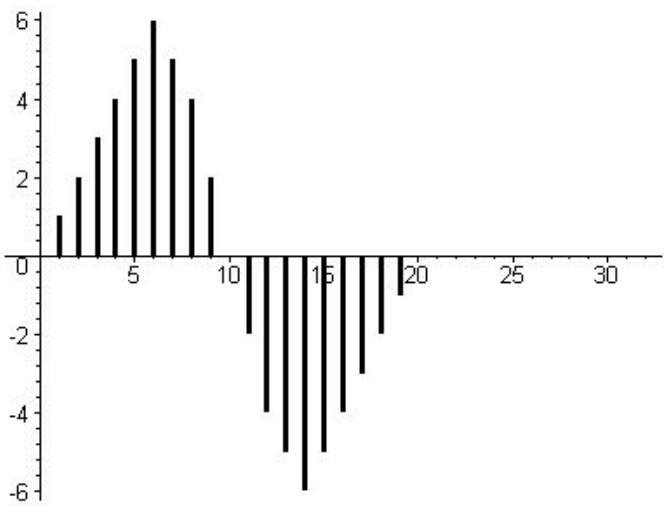

Figure 2. Coordinates of $w_{8}$.

$H_{k} \geqslant 4 m_{k}$ and $H_{k} \mid H_{k+1}$. We define $\sigma_{k}$ the following permutation of order $H_{k}$ : $\sigma_{k}=\left(1,2, \ldots, H_{k}\right)$; that is, $\sigma_{k}(i)=i+1$ for $1 \leqslant i \leqslant H_{k}-1$ and $\sigma_{k}\left(H_{k}\right)=1$. We enumerate the basis vectors of $Y$ as follows:

$$
\operatorname{span}\left(e_{n}, n \geqslant 3\right)=\operatorname{span}\left(e_{i, 1}, 1 \leqslant i \leqslant H_{1}\right) \oplus \operatorname{span}\left(e_{i, 2}, 1 \leqslant i \leqslant H_{2}\right) \oplus \ldots
$$

Therefore, each element $x$ of $X$ can be written

$$
x=x_{1} e_{1}+x_{2} e_{2}+\sum_{k=1}^{\infty} \sum_{i=1}^{H_{k}} y_{i, k} e_{i, k},
$$

and we put

$$
S x=x_{1} e_{1}+x_{2} e_{2}+\sum_{k=1}^{\infty} \sum_{i=1}^{H_{k}} y_{i, k} e_{\sigma_{k}(i), k} .
$$

$S$ has the following properties:

Lemma 4.3. i) $S$ is well defined and is an isometry.

ii) For $x \in X, \lim \left\|S^{H_{k}} x-x\right\|=0$. 
Proof. i) is because the norm is symmetric. For ii), it is enough to check it for $y \in Y$. Fix $\epsilon>0$. There exists an integer $N$ such that

$$
\left\|\sum_{l=N+1}^{\infty} \sum_{i=1}^{H_{l}} y_{i, l} e_{i, l}\right\| \leqslant \epsilon, \text { where } y=\sum_{l=1}^{\infty} \sum_{i=1}^{H_{l}} y_{i, l} e_{i, l} .
$$

For $k \geqslant N, H_{k}$ is a multiple of $H_{N}$, so for $1 \leqslant l \leqslant N$ and $1 \leqslant i \leqslant H_{l}, S^{H_{k}}\left(e_{i, l}\right)=$ $e_{i, l}$. Hence

$$
S^{H_{k}} y=\sum_{l=1}^{N} \sum_{i=1}^{H_{l}} y_{i, l} e_{i, l}+S^{H_{k}}\left(\sum_{l=N+1}^{\infty} \sum_{i=1}^{H_{l}} y_{i, l} e_{i, l}\right)
$$

and then

$$
S^{H_{k}} y-y=-\sum_{l=N+1}^{\infty} \sum_{i=1}^{H_{l}} y_{i, l} e_{i, l}+S^{H_{k}}\left(\sum_{l=N+1}^{\infty} \sum_{i=1}^{H_{l}} y_{i, l} e_{i, l}\right) .
$$

Since $S$ is an isometry, we get for $k \geqslant N:\left\|S^{H_{k}} y-y\right\| \leqslant 2 \epsilon$.

We now define the operator $R=R_{H S}$ (we use the same notation and assumptions as in the previous section for $F,\left(f_{k}\right)$ and $P$, and the definition of the sets $\tilde{A}_{R}$ and $\tilde{B}_{R}$ also remain unchanged). For $x \in X$, we put

$$
R x=S x+\sum_{k=1}^{\infty} f_{k}(P x) v_{k},
$$

where

$$
v_{k}=\epsilon_{k}\left(\sum_{i=1}^{m_{k}} e_{i, k}-\sum_{i=m_{k}+1}^{2 m_{k}} e_{i, k}\right) .
$$

The iterates of $R$ are given by (the same proof as in previous section)

Lemma 4.4. $R^{t} x=S^{t} x+\sum_{k=1}^{\infty} f_{k}(P x) v_{k, t}$ whenever $x \in X$ and $t \geqslant 1$, with $v_{k, t}=\left(I+S+\ldots+S^{t-1}\right) v_{k}$.

Now, let us choose the various constants: first, we introduce a sequence of positive and increasing integers $\left(a_{k}\right)$ such that

$$
\sum_{k=1}^{\infty} \frac{\left\|f_{k}\right\|}{a_{k}}<\infty \text { and } 1+a_{k} \mid 1+a_{k+1}
$$

Then, we put $m_{k}=a_{1} \ldots a_{k}, H_{k}=2\left(m_{k}+m_{k+1}\right)$. We have

$$
\frac{H_{k+1}}{H_{k}}=\frac{m_{k+1}+m_{k+2}}{m_{k}+m_{k+1}}=\frac{\prod_{i=1}^{k+1} a_{i}\left(1+a_{k+2}\right)}{\prod_{i=1}^{k} a_{i}\left(1+a_{k+1}\right)}=a_{k+1} \frac{1+a_{k+2}}{1+a_{k+1}},
$$

so we get $H_{k} \mid H_{k+1}$.

The constant $\epsilon_{k}$ is chosen such that $\left\|v_{k, 2 m_{k}}\right\|=1$. The finite-dimensional estimates give $\left\|v_{k}\right\| \leqslant \frac{2}{m_{k}}$. We deduce from this that $R$ is well defined and bounded because

$$
\left\|\sum_{k=1}^{\infty} f_{k}(P x) v_{k}\right\| \leqslant\left(\sum_{k=1}^{\infty} 2 \frac{\left\|f_{k}\right\|}{m_{k}}\right)\|P\|\|x\| \leqslant\left(\sum_{k=1}^{\infty} 2 \frac{\left\|f_{k}\right\|}{a_{k}}\right)\|P\|\|x\| .
$$

The following lemma is now a reformulation of the finite-dimensional estimates. 
Lemma 4.5. i) For fixed $k, v_{k, H_{k}}=0$ and the sequence $\left(v_{k, t}\right)_{t}$ is $H_{k}$-periodic.

ii) For $2 m_{k} \leqslant t \leqslant H_{k}-2 m_{k},\left\|v_{k, t}\right\|$ is maximal (in $t$ ) and $\left\|v_{k, t}\right\|=1$.

iii) For all $t,\left\|v_{k, t}\right\| \leqslant \frac{2 t}{m_{k}}$.

Proof. i) and ii) were proved before. For iii), use the triangle inequality and $\left\|v_{k}\right\| \leqslant$ $\frac{2}{m_{k}}$.

The proof of the theorem is now very close to the one we produced before. First, because the norm is symmetric and the $\left(v_{k, t}\right)_{k \geqslant 1}$ have disjoint supports, we get for $k \geqslant 1$ and $x \in X$ that

$$
\left\|\sum_{l=1}^{\infty} f_{l}(P x) v_{l, t}\right\| \geqslant\left|f_{k}(P x)\right|\left\|v_{k, t}\right\| .
$$

For each $t$, there exists $k$ such that $2 m_{k} \leqslant t \leqslant 2 m_{k+1}=H_{k}-2 m_{k}$. Thus Lemma 4.5 gives $\left\|v_{k, t}\right\|=1$, and we see that $\left\|R^{t} x\right\| \rightarrow \infty$ when $x \in \tilde{A}_{R}$. When $x \in \tilde{B}_{R}$, the proof is also in the same spirit as in section 3 (divide the sum into three parts), and if $\left|f_{k_{n}}(P x)\right| \rightarrow 0$, check that $R^{H_{k_{n}-1}} x \rightarrow x$ using Lemma 4.5. Details are left to the reader.

\section{FURThER RESUlTS AND REMARKS}

5.1. Difference between the operators. We denote by $\sigma(R)$ the spectrum of an operator $R$, by $\sigma_{p}(R)$ the set of its eigenvalues, by $r(R)$ its spectral radius, and we only consider complex spaces. Also let $\overline{\mathbb{D}}$ denote the unit closed complex disk and $\partial \mathbb{D}$ the unit complex circle. Let us recall that $\mathcal{A}_{X}$ is the set of operators which satisfy part A of Theorem 1.1 and that by Müller and Vršovský's result (see the introduction), if $\sum_{k=1}^{\infty} \frac{1}{\left\|R^{k}\right\|}<\infty$, then $A_{R}$ is dense. Suppose that $R \in \mathcal{A}_{X}$; then we must have $r(R)=1$. Indeed, if $r(R)<1$, by the spectral radius formula, $\left\|R^{k}\right\| \rightarrow 0$, which of course contradicts $A_{R} \neq \emptyset$. If $r(R)>1$, then if we fix $1<a<r(R)$, we get for $k$ large enough that $\left\|R^{k}\right\| \geqslant a^{k}$ (still with the spectral radius formula). Thus $\sum_{k=1}^{\infty} \frac{1}{\left\|R^{k}\right\|}<\infty$ and $A_{R}$ is dense, which is impossible. In particular, any operator $R \in \mathcal{A}_{X}$ has a spectrum contained in the unit closed disk, and its spectrum has to intersect the unit circle. More precisely, in the two studied examples (which are built with a strong analogy), we have:

Proposition 5.1. Assume that in the construction of the operator $R$ from section 3 , $\left(e_{k}\right)$ is a normalized Schauder basis; then $\sigma(R)=\left\{\lambda_{k}, k \geqslant 1\right\}$. On the other hand, if we take the operator $R_{H S}$ from section 4 , then $\partial \mathbb{D} \subset \sigma\left(R_{H S}\right) \subset \overline{\mathbb{D}}$.

Proof. Our operator can be written as $I+N$; thus we have $\sigma(R)=\{1+\lambda, \lambda \in$ $\sigma(N)\}$. Since $N$ is compact, $\sigma(N)=\sigma_{p}(N) \cup\{0\}$. Let $\lambda \in \sigma_{p}(N)$ and $x \neq 0, x=$ $\sum_{k=1}^{\infty} x_{k} e_{k}$ such that $N x=\lambda x$. Then

$$
\left\{\begin{array}{l}
0=\lambda x_{k} \text { for } k \in\{1,2\} \\
\left(\lambda_{k}-1\right) x_{k}+\frac{f_{k}(P x)}{m_{k-1}}=\lambda x_{k} \text { for } k \geqslant 3 .
\end{array}\right.
$$

If $\lambda \neq 0$, then $x_{1}=x_{2}=0$, that is, $P x=0$, and we get that $\lambda=\lambda_{k}-1$ for some $k \geqslant 3$. On the other hand, $N e_{k}=\left(\lambda_{k}-1\right) e_{k}$ for $k \geqslant 3$; hence $\sigma(N)=\sigma_{p}(N) \cup\{0\}=$ $\left\{\lambda_{k}-1, k \geqslant 1\right\}$ and $\sigma(R)=\left\{\lambda_{k}, k \geqslant 1\right\}$.

Now, the operator $S_{H S}$ of section 4 (associated to $R_{H S}$ ) has an infinite matrix which is a block diagonal with identity on the first block and then some matrices 
of Frobenius which have $X^{H_{k}}-1$ as characteristic polynomial on the $k$-th block (i.e. $\operatorname{span}\left(e_{i, k}, 1 \leqslant i \leqslant H_{k}\right)$ ). So by taking an eigenvector associated to a $H_{k}$ root of the unity for the matrix of Frobenius on the $k$-th block and completing it with some zeros, we see that

$$
G=\bigcup_{k=1}^{\infty} \mathbb{U}_{H_{k}} \subset \sigma_{p}\left(R_{H S}\right) \subset \sigma\left(R_{H S}\right),
$$

where $\mathbb{U}_{H_{k}}$ denotes the group of the $H_{k}$-th roots of the unity. Since $H_{k} \mid H_{k+1}, G$ is itself an infinite group of $\partial \mathbb{D}$ as a union of increasing groups. Thus $G$ is dense in $\partial \mathbb{D}$, and since $\sigma\left(R_{H S}\right)$ is a closed subset, we get $\partial \mathbb{D} \subset \sigma\left(R_{H S}\right)$.

The spectrum of $R$ is thus a sequence that goes very fast to 1 , whereas the spectrum of $R_{H S}$ is not countable.

Remark 5.2. With the notation above, we can show that if $F$ (the set defined on page 4$)$ is more than one line, then $0 \notin \sigma_{p}(N)$; that is, $1 \notin \sigma_{p}(R)$. Indeed, if $x=\sum_{k=1}^{\infty} x_{k} e_{k}$ is such that $N x=0$, then

$$
\left(\lambda_{k}-1\right) x_{k}+\frac{f_{k}(P x)}{m_{k-1}}=0 \text { for } k \geqslant 3 .
$$

If $P x \neq 0$, then since $\left|\lambda_{k}-1\right| \leqslant \frac{\pi}{m_{k}}$, we have

$$
\left|x_{k}\right| \geqslant \frac{\left|f_{k}(P x)\right| m_{k}}{\pi m_{k-1}} \geqslant \frac{15\left|f_{k}(P x)\right|}{\pi} .
$$

As $F$ is more than one line, we can find $\epsilon>0$ and a subsequence of $\left(f_{k}\right)$ with $\left|f_{k}(P x)\right| \geqslant \epsilon$. This is a contradiction because $\left|x_{k}\right| \rightarrow 0$ since $\left(e_{k}\right)$ is normalized, so $P x=0$ and $x=0$. If $F$ is just one line (which is a degenerated case for the operator $R$ ), then it is possible to have $0 \in \sigma_{p}(N)$. Indeed, if $F=\operatorname{span}\left(e_{1}\right)$, then by Proposition 2.1] $F \subseteq \operatorname{Ker} f_{k}$ for all $k$, so $f_{k}\left(e_{1}\right)=0$ and $N e_{1}=0$.

5.2. Size of the set $\mathcal{A}_{X}$. Now, we go back to a more general problem. We have shown that for any separable Banach space, $\mathcal{A}_{X}$ is non-empty. Hence, a natural question is: what can we say about the size of $\mathcal{A}_{X}$ ? It is clear that it cannot be dense for the norm operator because when $R \in \mathcal{A}_{X},\|R\|>1$. However, we have:

Proposition 5.3. If $X$ is a separable Banach space (or more generally satisfies the assumptions of Corollary 1.2), then the set $\mathcal{A}_{X}$ is dense for the strong operator topology on $\mathcal{L}(X)$.

To prove this, we shall use the following lemma [BM, p. 45].

Lemma 5.4. Let $X$ be a locally convex topological vector space, and let $R \in \mathcal{L}(X)$. Assume that for each integer $N$, one can find $x_{1}, \ldots, x_{N} \in X$ such that the vectors $x_{1}, \ldots, x_{N}, R\left(x_{1}\right), \ldots, R\left(x_{N}\right)$ are linearly independent. Then $\operatorname{Sim}(R)$ is SOT dense in $\mathcal{L}(X)$.

Proof of Proposition 5.3. Recall that the similarity orbit of an operator $R \in \mathcal{L}(X)$ is defined by

$$
\operatorname{Sim}(R)=\left\{J R J^{-1}, J \in \mathcal{G} \mathcal{L}(X)\right\}
$$

It is obvious to check that $\mathcal{A}_{X}$ is invariant under similarity: indeed, with the notations of Theorem 1.1, observe that if $S=J R J^{-1}$, then $x \in A_{R} \Leftrightarrow J x \in A_{S}$ and $x \in B_{R} \Leftrightarrow J x \in B_{S}$. Therefore, it is enough to prove the SOT density of $\operatorname{Sim}(R)$ 
for an operator $R \in \mathcal{A}_{X}$. For this, we will use our operator $R$ from section 3 (and Lemma 5.4). For example, we check it in the complex case. For $N \geqslant 1$, we put $x_{1}=e_{3}+e_{4}, x_{2}=e_{5}+e_{6}, \ldots, x_{N}=e_{2 N+1}+e_{2 N+2}$. Since $R e_{k}=\lambda_{k} e_{k}$ for $k \geqslant 3$, we see that for each $k,\left(x_{k}, R x_{k}\right)$ is a linearly independent family and that more generally $x_{1}, \ldots, x_{N}, R\left(x_{1}\right), \ldots, R\left(x_{N}\right)$ are linearly independent. Hence $\operatorname{Sim}(R)$, and therefore $\mathcal{A}_{X}$ is SOT dense in $\mathcal{L}(X)$. In particular, the set of operators which fail Prăjitură's conjecture is SOT dense in $\mathcal{L}(X)$.

On the other hand, we have:

Proposition 5.5. If $X$ is a complex Banach space, then the set of operators which satisfy Prăjitură's conjecture, that is,

$$
\mathcal{P}=\left\{R \in \mathcal{L}(X), A_{R}=\emptyset \text { or } A_{R} \text { is dense in } X\right\},
$$

is dense (for the norm operator) in $\mathcal{L}(X)$. In particular, $\mathcal{A}_{X}$ has empty interior.

Proof. Fix $\epsilon>0$ and $R \in \mathcal{L}(X)$. We can of course assume that $R \notin \mathcal{P}$. The arguments from the beginning of this section show that $r(R)=1$. Let $\lambda \in \sigma(R)$ with $|\lambda|=1$. Put $R_{\epsilon}=R+2 \epsilon \lambda I$; then $\left\|R-R_{\epsilon}\right\|=2 \epsilon$ and $\lambda(1+2 \epsilon) \in \sigma\left(R_{\epsilon}\right)$. Hence $r\left(R_{\epsilon}\right) \geqslant 1+2 \epsilon$, and by the spectral radius formula, we get that for $k$ large enough $\left\|R_{\epsilon}^{k}\right\| \geqslant(1+\epsilon)^{k}$, so $\sum_{k=1}^{\infty} \frac{1}{\left\|R_{\epsilon}^{k}\right\|}<\infty$ and $R_{\epsilon} \in \mathcal{P}$ because $A_{R_{\epsilon}}$ is dense in $X$ by Müller and Vršovský's theorem.

\section{ACKNOWLEDGEMENTS}

The author would like to thank his advisor, Robert Deville, for suggesting this topic and for his help during the elaboration of this paper; Luis Sanchez for fruitful conversations; and the anonymous referee for useful comments.

\section{REFERENCES}

[AH] S.A. Argyros and R.G. Haydon, An $\mathcal{L}^{\infty}$ HI space solving the $\lambda I+K$ problem, Preprint. http://arxiv.org/abs/0903.3921

[BM] F. Bayart and E. Matheron, Dynamics of linear operators, Cambridge Tracts in Mathematics (2009). MR2533318 (2010m:47001)

[GM] W.T. Gowers and B. Maurey, The unconditional basic sequence problem, J. Amer. Math. Soc. 6, 851-874 (1993). MR.1201238 (94k:46021)

[HS] P. Hájek and R.J. Smith, Operator machines on directed graphs, Integral Equations Oper. Theory 67, no. 1, 15-31 (2010). MR.2629974

[MV] V. Müller and J. Vršovský, On orbit-reflexive operators, J. London Math. Soc. 79, 497-510 (2009). MR2496526 (2010b:47021)

[OP] R.I. Ovsepian and A. Pelczynski, On the existence of a fundamental total and bounded biorthogonal sequence in every separable Banach space, and related constructions of uniformly bounded orthonormal systems in $L^{2}$, Studia Math. 54, 149-159 (1975). MR0394137 $(52: 14942)$

[P] G.T. Prăjitură, The geometry of an orbit. Operator theory live, 145-154, Theta Ser. Adv. Math., 12, Theta, Bucharest, 2010.

[R] C. Read, The invariant subspace problem for a class of Banach spaces. II. Hypercyclic operators, Israel J. Math. 63, 1-40 (1988). MR.959046 (90b:47013)

[T] L. Tzafriri, On Banach spaces with unconditional bases, Israel J. Math. 17, 84-93 (1974). MR.0348456(50:954)

Department of Mathematics, Université Bordeaux 1, 351, cours de la Libération, F-33405 TAlence Cedex, France

E-mail address: jean-matthieu.auge@math.u-bordeaux1.fr 